\title{
Flexible Infrastructure for Virtual Enterprises
}

\author{
A.T.M. Aerts, N.B. Szirbik, J.B.M. Goossenaerts \\ Eindhoven University of Technology, Eindhoven, The Netherlands \\ email: A.T.M.Aerts@tue.nl, \{N.B.Szirbik, J.B.M.Goossenaerts\}@tm.tue.nl
}

Keywords Virtual Enterprise, Infrastructure, Flexibility, Mobile Agents

\begin{abstract}
In recent papers we studied the use of a mobile agent framework to support the primary process in a Virtual Enterprise. We argued that the installation of standard software modules, called service bridges or docks, at the participant enterprises provides a suitable infrastructure for the use of mobile agents. The deployment of mobile software agents using such modules has been studied in applications such as networked electronic trading and mediation of negotiations. The emphasis in these applications lies on demonstrating the potential of mobile agents for the support of complex decision problems. As such they focus on the agent - agent interaction. In this paper we study the agent - system interaction. We will discuss a number of change cases and examine their impact on the requirements on the agent - agent and on the agent - system interaction arising from the need for flexibility.
\end{abstract}

\section{INTRODUCTION}

A Virtual Enterprise (VE), in our view, is a conglomerate of regular enterprises that collaborate on an ad hoc basis. The term was derived from "Virtual Organisation" [5], which denotes a group of people from different organisational structures. These people usually have a common short-term goal, and they form for a short period a team that can be viewed as an organisational structure, which crosses the boundaries of the long-term organisations they belong to. Similarly, a VE is an ad hoc organisation that responds to unexpected change, for example to one-of-a-kind business opportunities, such as the one-time production of a specific landing gear in the case of some unexpected damage, a batch of mobile phones as part of some promotional activity, or some emerging niche market. Of course, the enterprises that participated in the production of one particular item will know how to find each other when a similar opportunity arises. The result 
will be a group of enterprises, with complementary capacities, that can collaborate on the production of a set of related products, such as aircraft subsystems, whose complexity transcends the abilities of a single enterprise. Early examples of virtual enterprises include the nineteenth century whaling industry and nowadays the movie industry (for a history of the VE concept see [8]).

Characteristic for the members of a VE nowadays is that they commit only a minor part of the entire production capacity to the VE (see Figure 1). The major part is committed to long-term alliances, e.g., to one or more supply chains in which they are a link.

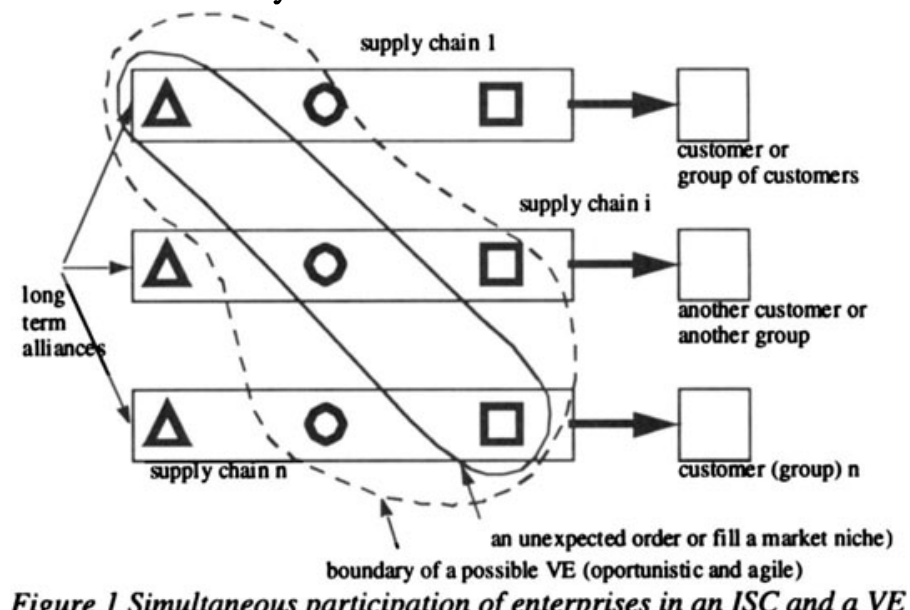

\subsection{VE and Integrated Supply Chain}

Enterprises use the VE strategy to meet unexpected change and unforeseen events (i.e., become agile). One of the beneficial results is that unused capacities or planned over-capacity can be made productive. To cope with momentary unavailability of a particular type of capability, a VE will include several members with similar capabilities (redundancy). This is opposed to the concept of lean structure, but it will help the VE itself to be agile [8]. In view of the opportunistic nature of the business, the integration of the ICT systems of the participants will be at the operational level, limited to the exchange of data on availability, prices and products. These characteristics distinguish the VE from a more long-term inter-organisational structure, such as the Integrated Supply Chain (ISC). An ISC is a lean, stable business organisation that caters to a relatively stable market. Usually, such an organisation is designed to cope with foreseeable changes in supply and demand. There are stable procedures for fast global re-planning and local rescheduling, which make the organisation flexible, but it is not agile, since it will have difficulty coping with unexpected change. The ICT integration in a 
Supply Chain is at a higher level, involving also the exchange of forecasts and production schedules, and the capacities are committed for a longer period of time. This level of integration is needed to provide the necessary performance in terms of delivery time and costs.

\subsection{VE and e-Business}

A VE is also distinct from an e-Business, which typically uses the World Wide Web as an additional communication channel to reach clients. Two new interesting examples of e-Businesses, which among other things address the problem of providing aircraft subsystems, are Aeroxchange [1] and MyAircraft [11]. Both companies define a digital marketplace for aerospace parts and components, where parts can be leased, bought, sold or auctioned.

In addition, services are offered to facilitate the exchange of scheduling and replenishment planning information, so that both customers and suppliers can optimise their supply chains. These services may range from supply chain management, including collaborative demand forecasting and supply planning, to trading and e-procurement, including order promising, and vendor managed inventories. To facilitate the usage of these services system integration support is offered. Both virtual companies are run from a central website that allows all parties involved (customers, suppliers) to selectively communicate with each other. A specific feature of both is that the provider of the software is a separate and highly specialised company, Oracle for Aeroxchange and $\mathrm{I} 2$ for MyAircraft.

In this paper we will take the point of view that the VE has been formed. A discussion of the formation process can be found, e.g. elsewhere in these proceedings [15].

\section{MOBILE AGENT INFRASTRUCTURE}

Enterprises can join or leave the VE at short notice, depending on capability and opportunity. The volatility of virtual enterprises imposes strong requirements on their ICT support. In order for the VE to be agile, the ICT infrastructure must be highly flexible. Typical events that a VE has to be resilient to are:

- An enterprise joins the VE.

- An enterprise leaves the VE.

- The VE expands its catalogue with new products.

- The VE merges with another VE to enter a new market. 


\subsection{Mobile Agent Support for the VE primary process}

One way to provide the required flexibility is to use a mobile agent framework. The deployment of mobile software agents has been studied in applications such as networked electronic trading [4] and mediation of negotiations [13]. In the latter, software agents play the role of a human supervisor, who is in charge of the tracking, monitoring and problem management of a specific product item. This is inspired from real business cases, where rush orders are assigned a special manager to supervise their fulfilment. The Mobile Agent System can be envisaged as a Support System for this human manager. When a customer places an order, e.g. via a Web portal, which is accepted, an agent is made responsible for filling the order. The agent monitors the assembly of the ordered product and sends out agents to provide the necessary components. These agents move to those enterprises in the VE that have the capacity to deliver the required components at the right time. If an enterprise needs components from other enterprises to produce its own component another batch of agents is sent out to supervise the delivery of these components.

The agents are programmed to perform the monitoring task, and also handle exceptions. For instance, it is possible that one of the enterprises in the VE cannot commit, for whatever reason, production capacity that it had previously advertised as available. The agent that comes to claim this capacity finds that it is no longer available and has to find another enterprise in the VE to provide the required capacity. This will of course entail some negotiations, e.g., to free the required capacity at the right time and arrange compensation. The agents take care of routine tasks, including negotiations, and involve the human decision maker in more complicated or unforeseen situations.

\subsection{Mobile Agent Framework requirements}

A Mobile Agent framework has to satisfy a number of properties to provide a suitable infrastructure [9]. In [2] we argued that specification of agent behaviour by means of rules makes it possible to change to behaviour by changing some of the rules. Given an appropriate rule system this could perhaps even be done by the decision maker the agent has to support.

Deployment of the agent infrastructure should also be easy. A party that wants to join a fairly volatile organisation such as a VE has a low level of commitment and should not be required to invest a lot of time and effort to be able to join. In recent papers we studied the use of a mobile agent framework [7] to support the primary process in a Virtual Enterprise [2]. We argued that the installation of standard software modules, called docks and service bridges at the participant enterprises provides a suitable 
infrastructure for the use of mobile agents. At the dock, agents can be created, and sent out, and they can come back there to communicate with the local information system. Since the docks usually are available on several platforms, they shield the agent environment from hardware and OS heterogeneity. The Internet provides the basic connectivity. The service bridge provides the semantic alignment of the local IS to the common shared ontology.

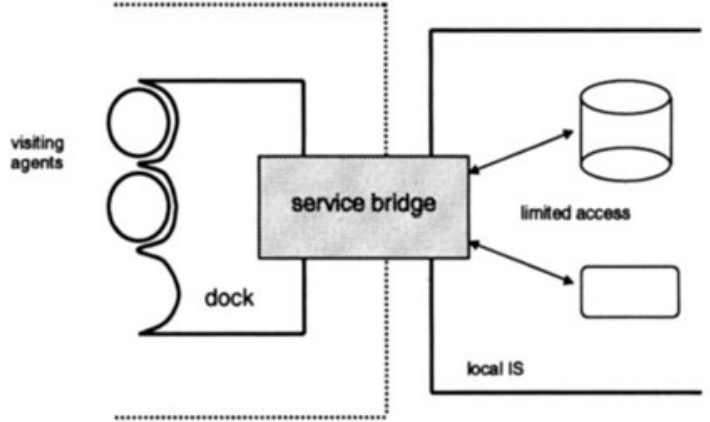

Figure 2 Architecture of the dock and service bridge component

On top of the basic infrastructure the VE support system can be built and consists of the mobile agents that provide the product tracking functionality [13]. In addition, some components, such as the web portal, with a product catalogue and ordering and tracking facilities that serve the VE as a whole are needed, and some components that are specific to an individual enterprise.

\subsubsection{VE-level components}

In [2] it was argued that in addition to a shared interface to the customers (e.g., a Web-portal), also other services, such as a scheduling service, should be made available at the VE-level, i.e., to the monitoring agent system as a whole. The scheduling service determines whether it is possible to fill the order and allocates the proper enterprises to the order. The scheduler needs data from the enterprises about availability of ready-made components or production capacities, information that may be gathered on demand by special roaming agents. An alternative is to store the information in a central repository and refresh it on a regular basis. When customer orders are incidental, such as those arising from a broken down landing gear, the first approach may be sufficient. But one can also envisage Virtual Enterprises that cater to emerging markets and have to deal with a higher frequency of 
ordering. In such a case a central body of availability data is more appropriate and moreover, it can serve as a basis for order promising.

The agents should be able to communicate amongst themselves and with the local information systems. The former requires a shared perspective, such as a common ontology, which would detail the representation and semantics of data about, e.g., availabilities, bills of material and routing, and procedures to be followed, e.g., in the case of negotiations. A lot of work on standardization of this kind of information has been done in the context of electronic data interchange (EDI) by large car and aircraft manufacturers, and many others, to organize their supply chains. Also, platform organisations in the domains of, e.g., commerce, health care and transportation have made similar efforts. This implies, that in many areas already the knowledge and experience exists, that is needed to establish a common ontology. Recently, two new developments have taken place in this area. The first is EDI via Internet that will allow also smaller businesses to partake in EDI at a lower cost of investment than would be required to obtain EDI-services from a particular Value Added Network (VAN). Because of this, also participation in a VE becomes quite feasible for these kinds of businesses. The second is the introduction and fast adoption of XML to make the messages self-descriptive. This will add a flexibility of operation, not found in the often proprietary, extensive and rigid EDI-message frameworks. Given the ontology, the agents can communicate among themselves using an agent communication language $[6,10]$. A service at the VE level would be to provide this ontology to new VE members.

\subsubsection{Enterprise-level components}

An enterprise that will join a VE will have to conform to the VE common ontology and map their internal concepts and procedures to the common counterparts. This requires components that are specific to an enterprise since they involve the integration of the information systems of the enterprise with the VE support system. These components reside at the service bridges. In fact, a service bridge (see Figure 2) can be seen as consisting of two parts, connected by a gateway. One part is external to the enterprise and belongs to the general infrastructure of the VE. It is the same at every site and provides the mobile agents with a uniform facility to come and visit the site. This part is, for security reasons, continuously verified for code consistency in order to make sure, that it has not been tampered with by malicious visiting agents. The second part is internal to the enterprise and connected to the external part via a secure gateway. Here the mapping between the VE ontology and the enterprise's internal ontology is executed, 
the access to the enterprise data and communication with systems or parties in the enterprise is controlled.

\section{VE ORGANISATION AND ROLES}

\subsection{The role of the mobile agents}

The role of the agents is to extend the local information systems with mobile functionality to enable enterprises to participate in the VE business process. The advantage is a reduction of the local effort an enterprise has to make to join the VE. Moreover, a change in the business process can be dealt with, as far as support functionality is concerned, at the agent level (e.g., by upgrading the agent rule set).

\subsection{The role of the software agent provider}

The perceived volatility of the VE poses a curious problem with regard to the provision of the required infrastructure: should the VE develop and maintain its infrastructure (docks, agents, global services) itself? This proposition implies that some enterprises in the VE will have a more than average commitment to the VE. Should one of these leave, then the continuation of the VE may be in danger. Our position is that the development of the agents and the common services, such as scheduling and repositories for data and the ontology is best handled by an independent, trusted third party, that we call SACP (Software Agent Common Provider) [13]. This SA-provider plays a role similar to the Internet-, the VAN- and the ASP-providers. They all offer communication and application services to customers at various levels of performance, security and reliability. They also take care of the development and maintenance of required software. Since they will provide services to more than one VE and solutions can be reused, the costs will be lower and also the threshold in terms of required technology, knowledge, and investments for smaller companies to join will be lower.

An independent third party is also a guarantee for the autonomy of the participating enterprises, especially of the smaller ones. In the context of extended enterprises [3], mobile agents have been considered as a lightweight extension of the dominant company's ERP system to its suppliers. The result was a tight integration of the supplier's information system into that of the extended enterprise. Such a lock-in allowed the dominant company to manipulate the supplier's production and delivery schedules to optimise the performance of the extended enterprise. This effectively blocks a similar collaboration of the supplier with other customers. 
We see that in the case of MyAircraft and Aeroxchange some parties have so large a commitment that they have invested in a joint venture to deal with not just incidental, but also the stable provisioning of aircraft parts and services, aimed at supply chain optimisation. These ventures clearly have a more long-term perspective. An infrastructure has been developed with a number of global (in this case even central) services, and with a low threshold (for a number of services a Web browser is sufficient) for other companies to participate. In a VE, one-of-a-kind orders are predominant. To make the investment into VE-level services worthwhile, the confidence has to exist among a number of parties, that more opportunities will present themselves. The possible reuse of the infrastructure for similar products (viz. small changes in the Bill of Material) then is an important requirement.

\subsection{The role of the participants}

The role of the participants in the VE is to make their production capacities available to the VE. The SACP can assist in building the software needed to transform between the enterprise and the VE data and procedures, by supplying documentation, templates, and even help-desk support. Many back-end systems already offer connectivity support, ranging from JDBCinterfaces and Business connectors to Business connectivity. The final control should stay with the participant to increase the level of confidence in and acceptance of the VE system. The participant should determine what kind of data and IS functions to expose to the VE (see Figure 2).

\section{CHANGE CASES}

\subsection{An enterprise joins the VE}

In the previous section we discussed some of the infrastructure needs a VE. The first step for an enterprise that wants to join the VE then is to become familiar with the VE ontology. The VE should therefore offer a mechanism whereby an enterprise can express its intention to join and, upon admission, get access to the necessary information, such as the VE ontology. The VE-Web portal can provide this functionality. As a second step, the prospective participant has to publish its resource types and availabilities to the VE. To achieve this, the prospective participant should install a dock and service bridge and connect it to its back-office systems by implementing the translation methods (semantic alignment) to and from the VE ontology. When the necessary communication channel is established, the enterprise can be included in the list of members of the VE. Agents can now visit the new participant, and the VE-mechanism for updating the availabilities can be used to also include the capacities of the new participant. 


\subsection{The VE expands its catalogue with new products.}

The addition of a new participant by itself only increases the redundancy of the VE. However, a new participant may also possess production capabilities of a type that was not available to the VE before and open up the possibility for new products. A similar situation can arise when an enterprise wants to expand the list of capabilities that it offers to the VE with a capability not yet available to the VE. At this point the VE must be aware of the opportunity to expand its catalogue. This can be done in several ways.

The initiative for this action can be taken by the enterprise that is offering the new capability and wants to make it more productive. This enterprise has to consult the VE-ontology and compare it to its own product catalogue. When the enterprise catalogue contains products that in addition to the new capacity only require production capacities available in the VE, the enterprise can propose to the VE to include these products in its catalogue. Proper procedures to do this have to be set up.

Another trigger for the expansion of the catalogue may come from customers who inquire if the VE can produce a product not in the catalogue. The VE in this case should provide a facility by which a customer can specify a product, complete with the required resources. The customer does not necessarily use the same ontology as the VE does, and so support to produce the correct specification may be needed. Given the product specification, the VE can check to see if the required capacities are available (even if they have not yet been in use), or, if some capabilities are missing, inquire among its participants to see if any may be willing to offer these capabilities. In the case of a positive answer the capacities may be added to the list of VE-resources and the new product may be included in the VE catalogue.

Expansion of the VE catalogue of products has an impact on the ontology. When the addition to the catalogue concerns variants of previous offerings the impact may be minor, just affecting a (part of a) bill of material (BOM) or routing. Flexibility in the agent communication now requires that the BOM can be passed as a parameter (for example, in a self describing format as XML) so that variations in composition pose no problem. When the new product involves new concepts, such as production techniques that are new to the VE also the ontology has to be expanded.

\subsection{An enterprise leaves the VE}

When an enterprise leaves the VE, the inverse process of a new member joining and a new product being added to the catalogue has to be carried out. The enterprise has to make its withdrawal known to the VE. The VE then 
has to check whether the withdrawing enterprise has unique production capabilities. If not, then all that has to be done is to remove that enterprise's capacities from the set of available resources. Only the redundancy of the $\mathrm{VE}$ is affected in this case. When the enterprise was offering unique capabilities, also those products have to be removed from the catalogue, for which the contribution of the leaving participant is essential.

\subsection{The VE merges with another VE to enter a new market}

The possibility of a merger between two VE's is a feasible option when there already is considerable over-lap between the activities and capacities of two, e.g. one is specialized in mobile phones, and the other in PDA's, i.e. devices, which have a number of components in common. This implies that some enterprises will be a member of both VE's. The merger scenario is simplest, when both VE's are using the same infrastructure (obtained from the same SACP). In this case, a new VE can be set up by combination of infrastructures. The ontologies must be merged, which probably will require some semantic alignment. Once that is done also the catalogues and lists of resources can be combined. The next step then is the expansion of the catalogue to exploit the enhanced production facilities. In case the two VE's do not use the same infrastructure, the merger will have to be effected by adding the members of one VE to the other. This procedure requires the repeated application of the scenarios discussed in par. 4.1 and 4.2.

We conclude from the use cases above that the VE, in addition to providing flexible support for the production process itself, also will have to provide a number of services, such as an ontology server and capability and product addition and removal services, that will support the dynamics of the composition of the VE. These services rely on sufficiently expressive specification languages and sufficiently widely accepted standards.

\section{CONCLUSION}

The infrastructure presented here differs from more specialized system integration architectures such as federations [12] and mediators [14], which also provide a unified view of the system to the outside world (user or application). In both architectures, the transformation to the local representations is done centrally, so that the local systems remain unaffected. This, however, requires the local systems to publish a suitable part of their interfaces to the global system, implying a more long-term commitment. Both architectures require synchronous communication with their components and are better suited for local area network environments. Of 
course, the infrastructure proposed in this paper can be specialized for the support of federation or mediation type architectures.

The agent infrastructure discussed in this paper can also be applied in other situations. In addition to supporting the VE strategy it can be used in ecommerce situations, such as auctions and in supply chains. In the case of auctions, no integration with the back-office systems is required. The agents provide in this case an intelligent interface that allows a customer to specify, e.g. a bidding strategy.

In the case of the integrated supply chain, the agents may provide a more flexible alternative to the EDI-procedures in use at present. When the agents are supplied with error handling functionality to autonomously solve a number of common problems, the system of procedures and messages may be significantly simplified. When suitably extended, the agent framework discussed in this paper therefore may be used in e-market and supply chain situations as well [16].

The mobile agent system will be tested in some small-scale experiments in the PROVE project $[2,13]$.

\section{REFERENCES}

[1] Aeroxchange, http://www.aeroxchange.com

[2] Aerts, A.T.M., Szirbik, N.B., Hammer, D.K., Goossenaerts, J.B.M., Wortmann, J.C. (2000) "On the Design of a Mobile Agent Web for supporting Virtual Enterprises ", WET ICE 2000.

[3] Browne J., Sackett P.J., Wortmann J.C., (1995), "Future manufacturing systems Towards the extended enterprise", Computers in Industry, 25, Elsevier, pp.235-254.

[4] Dasgupta, P., Narasimhan, N., Moser, L.E., Melliar-Smith, P.M., (1999), "Mobile Agents for Networked Electronic Trading", in IEEE Transactions on Knowledge and Data Engineering, vol. 11, no. 4, pp.509-525.

[5] Davidow W.H., Malone M.S., (1992), "The Virtual Corporation", Harper and Collins, New York.

[6] The Foundation for Intelligent Physical Agents, "FIPA99 language Specification", http://www.fipa.org

[7] Goossenaerts, J.B.M., Aerts, A.T.M., Hammer, D.K., (1998), "Merging a Knowledge Systematisation Framework with a Mobile Agent Architecture", in Information Infrastructures for Manufacturing II, (Mills \& Kimura, eds.), Kluwer, The Netherlands.

[8] Goranson, H.T., (1999), "The Agile Virtual Enterprise: case, metrics, tools", Quorum Books, Westport.

[9] Hammer D.K., Aerts A.T.M., Dalmeijer M., (1998), Mobile Agents Architectures: What are the design Issues? International Conference on Engineering of Computer Based Systems (ECBS), Jerusalem, Israel, March 1998.

[10] Labrou Y., Finin T., (1997), “A proposal for a New KQML specification”, Technical Report CS-97-03, Computer Science and Electrical Engineering Dept., University of Maryland, Baltimore.

[11] MyAircraft, http://www.myaircraft.com 
[12] Sheth, A.P., Larson, J.A., (1990), “Federated Database Systems for managing distributed, heterogeneous and autonomous databases", ACM Computing Surveys, 22(3), 183-236.

[13] Szirbik, N.B., Wortmann, J.C., Hammer, D.K., Goossenaerts, J.B.M., Aerts, A.T.M., (2000), "Mediating Negotiations in a Virtual Enterprise via Mobile Agents", AIWoRC'2000: Mobile Technologies and Virtual Enterprises", Buffalo.

[14] Wiederhold, G., (1994), "Interoperation, Mediators and Ontologies", Proc. Int. Symp. On Fifth Generation Computer Systems, Vol. W3, 33-48, ICOT, Tokyo.

[15] Wiendahl, H.-P., Engelbrecht, Hamacher, O., (2000), "From Single Enterprises to Complementary Networks", Proceedings of DIISM2000, the 4th International Conference on Design of Information Infrastructure Systems for Manufacturing 2000, 15-17 November 2000, Melbourne, Australia.

[16] Wortmann J.C., Szirbik N.B., (2000), "ICT Issues among Collaborative Enterprises: from Rigid to Adaptive Agent-Based Technologies", to be published in Intl. Journal of Production Planning and Control. 